\title{
A targeted reweighting method for accelerating the exploration of high- dimensional configuration space
}

Cite as: J. Chem. Phys. 123, 234908 (2005); https://doi.org/10.1063/1.2137704

Submitted: 10 August 2005 . Accepted: 18 October 2005. Published Online: 20 December 2005

R. I. Cukier, and M. Morillo

\section{ARTICLES YOU MAY BE INTERESTED IN}

Accelerated molecular dynamics: A promising and efficient simulation method for biomolecules

The Journal of Chemical Physics 120, 11919 (2004); https://doi.org/10.1063/1.1755656

A statistical analysis of the precision of reweighting-based simulations

The Journal of Chemical Physics 129, 034103 (2008); https://doi.org/10.1063/1.2944250

Sampling of slow diffusive conformational transitions with accelerated molecular dynamics

The Journal of Chemical Physics 127, 155102 (2007); https://doi.org/10.1063/1.2789432
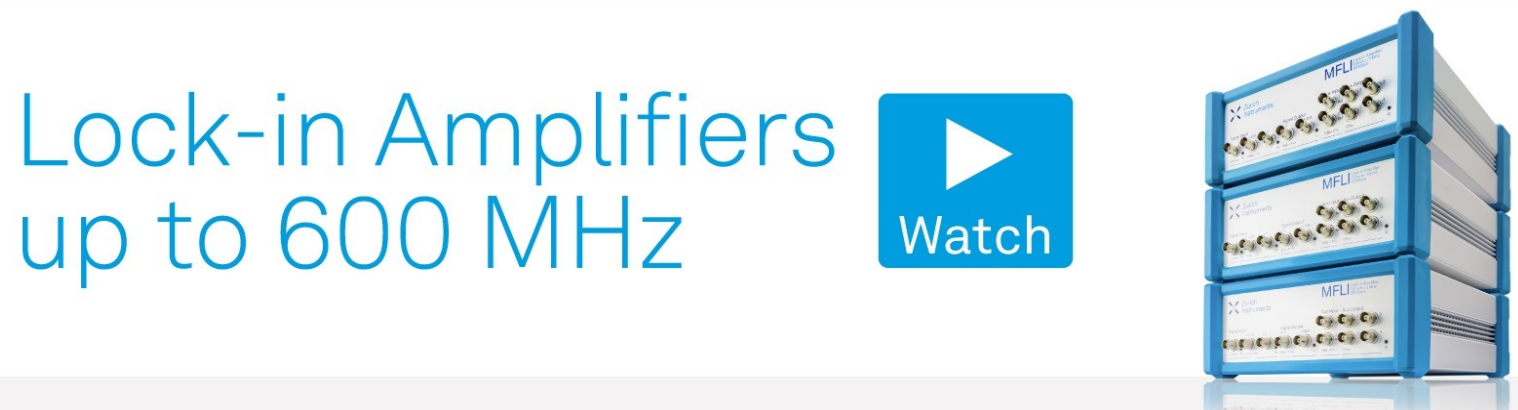

J. Chem. Phys. 123, 234908 (2005); https://doi.org/10.1063/1.2137704 


\title{
A targeted reweighting method for accelerating the exploration of high-dimensional configuration space
}

\author{
R. I. Cukier ${ }^{\text {a) }}$ and M. Morillo ${ }^{\text {b) }}$ \\ Department of Chemistry, Michigan State University, East Lansing, Michigan 48824
}

(Received 10 August 2005; accepted 18 October 2005; published online 20 December 2005)

\begin{abstract}
Time scales available to biomolecular simulations are limited by barriers among states in a high-dimensional configuration space. If equilibrium averages are to be computed, methods that accelerate barrier passage can be carried out by non-Boltzmann sampling. Barriers can be reduced by modifying the potential-energy function and running dynamics on the modified surface. The Boltzmann average can be restored by reweighting each point along the trajectory. We introduce a targeted reweighting scheme where some barriers are reduced, while others are not modified. If only equilibrium properties are desired, trajectories in configuration space can be generated by Langevin dynamics. Once past a transient time, these trajectories guarantee equilibrium sampling when reweighted. A relatively high-order stochastic integration method can be used to generate trajectories. The targeted reweighting scheme is illustrated by a series of double-well models with varying degrees of freedom and shown to be a very efficient method to provide the correct equilibrium distributions, in comparison with analytic results. The scheme is applied to a protein model consisting of a chain of connected beads characterized by dihedral angles and the van der Waals interactions among the beads. We investigate the sampling of configuration space for a model of a helix-turn-helix motif. The targeted reweighting is found to be essential to permit the original all-helical conformation to bend and generate turn structures while still maintaining the alpha-helical segments. () 2005 American Institute of Physics. [DOI: 10.1063/1.2137704]
\end{abstract}

\section{INTRODUCTION}

Biomolecular processes encompass a large range of space and time scales, from local subpicosecond motions to large-scale domain motions that may extend to seconds. ${ }^{1,2}$ These processes take place in a high-dimensional configuration space and present a challenge to simulation methods. The issue arises in studies relevant to protein folding, ${ }^{3-5}$ large-scale functional domain motions in response to various stimuli, ${ }^{6}$ the entry of substrates into active sites and the resulting mutual accommodation of protein and substrate, ${ }^{7}$ and the local rearrangements of protein and substrate in response to various steps in the chemical transformations of enzymes. ${ }^{8}$ The origin of the difficulty is in the large variety of barriers to motion that must be overcome to sample properly the configuration space. These barriers are a result of the rough energy landscape of proteins and lead to trapping of the configuration point in local minima on the energy surface. ${ }^{9}$ If the simulation methods cannot overcome these barriers (repeatedly) in the available simulation time, improper configurational sampling will occur and false conclusions may be reached.

If the desire is equilibrium (Boltzmann) sampling, versus exploration of dynamic phenomena (for example, time correlation functions) then a number of methods are possible that modify the system's equations of motion that, while pre-

\footnotetext{
${ }^{\text {a) }}$ Author to whom correspondence should be addressed. Fax: (517)-3531793. Electronic mail: cukier@cem.msu.edu

${ }^{b)}$ Permanent address: Fisica Teorica, Universidad de Sevilla, Apdo, Correos 1065, Sevilla 41080, Spain.
}

cluding correct dynamics, will still lead to Boltzmann sampling. Free-energy methods are based on this trade-off. If a (low-dimensional) reaction coordinate is known, or is a reasonable approximation, then there are a number of biasing methods that can construct the potential of mean force along this reaction coordinate. ${ }^{7,10-15}$ The umbrella sampling method, ${ }^{10}$ for example, adds artificial potentials that emphasize desired regions of configuration space and permits construction of a potential of mean force along a desired reaction coordinate. If there is a barrier, then rates of transitions may be estimated by transition state theory. ${ }^{16}$ If stable wells separated by free-energy barrier energies are found, then reduced descriptions such as kinetic equations can be formulated with the stable states as the species and the rate constants in the model parametrized by transition-state-theory expressions. ${ }^{17}$

When a reaction coordinate is not singled out, or known, then focus shifts to enhancing and accelerating the exploration of the configurational space by various generalized ensemble (non-Boltzmann-sampling) schemes. Examples are multicanonical ${ }^{18-23}$ and parallel tempering (replica exchange $)^{19,22-26}$ methods that attempt to surmount barriers without an explicit focus on particular degrees of freedom. A difficulty with generalized ensemble methods is the lack of knowledge of what the weighting should be; it must be estimated "on the fly." Another method that does directly determine the weight factor, "stochastic tunneling,"27 was introduced and explored in several directions, including protein folding, ${ }^{26}$ receptor-ligand docking, ${ }^{28}$ and exploration of dihedral conformational space in the alanine dipeptide. ${ }^{29}$ Hamelberg et $a l^{29}$ smooth the potential-energy surface by raising 
up the minima. They maintain the underlying shape of the unmodified potential-energy surface by smoothly merging with the original potential at a threshold energy value, and by using a two-parameter scaling. A similar strategy was pursued by Liu and Berne in the context of equilibrating a Lennard-Jones fluid and a model polymer. ${ }^{30}$ In that work, the potential is switched between normal and lowered-barrier forms according to a probabilistic rule. The idea in these methods is to modify the known potential-energy surface in a definite way, with the consequence that barriers in the explicit terms in the surface are reduced and thereby the configuration-space exploration may be accelerated. To compute equilibrium averages, the trajectory generated by this modified surface is corrected by a "reweight" factor that serves to restore the correct Boltzmann weighting.

In this article, we pursue an approach based on modifying selected terms in the potential-energy function used in the dynamical equations. We will refer to the method as targeted reweighting. In our view, scaling the overall potential (global scaling) will not in general be successful; indeed, it may well be counterproductive if it forces the system to spend a great fraction of the trajectory exploring unphysical regions of configuration space. While, in principle, the reweighting step will correct for the modified trajectory, the scheme will be computationally inefficient. For a given barrier $V_{b}$ the relative acceleration in surmounting it based on the potential $V$ and its scaled $g V(0<g<1)$ version is, according to transition state theory, $\exp \left(-(g-1) \beta V_{b}\right)$, where $0<g<1$. The exponential character indicates a large enhancement in the rate of barrier passage. However, if taken to the extreme, the sampling would become uniform, and the search space would grow exponentially. With a uniform scaling factor $g$, some terms would be properly scaled, but others would be overscaled and lead to overexploration of configuration space. Thus, for a given potential barrier, a compromise scaling must be used, and the reweighting should be targeted to the terms in the potential-energy function that are responsible for the difficulties in rapidly accessing important parts of configuration space. The works of Hamelberg et al. ${ }^{29}$ and Liu and Berne also selectively target certain degrees of freedom. $^{30}$

Once interest shifts to equilibrium sampling, versus generating correct dynamics, the possibilities for the equations of motion that can sample the equilibrium distribution are enhanced. Given a potential-energy function $V\left(q^{N}\right)$ of a set of generalized coordinates $q^{N}$, at any level of description, for example, fully atomistic as in the typical moleculardynamics or Monte Carlo force fields, ${ }^{11,31}$ or in a reduced dihedral coordinate space, all that must be guaranteed is that sampling is obtained according to the Boltzmann distribution $\sim \exp \left(-\beta V\left(q^{N}\right)\right)$ with $\beta=1 / k_{B} T$. Here, we will use a Langevin dynamics $^{32,33}$ in configuration space that will produce the correct Boltzmann weighting. The temperature is introduced via the correlation function of the noise term in the Langevin equation. The form of the Langevin equation suggests the use of an integrator, developed by Helfand ${ }^{34}$ and Greenside and Helfand, ${ }^{35}$ which is a stochastic generalization of the fourth-order Runga-Kutta integrator. The order of this inte- grator permits the use of relatively large time steps, and its introduction to these force-field-directed problems should be of general utility.

The targeted reweighting is first explored in the context of a simple model, where the equilibrium distribution (though not the dynamics) is available analytically. It consists of a set of $N$ double wells, each separated by a barrier. The state space here scales as $2^{N}$ and has the character of a rapidly increasing entropic problem if the scaling is done too heavily. When the double wells are asymmetric, they can evoke the resolution of the Levinthal paradox ${ }^{36-38}$ that suggests that without some downhill bias a protein would not be able to fold on a practical time scale, due to the exponentially large configuration space. As a more realistic (though not analytically tractable) example, we use a model introduced by $\mathrm{He}$ and Scheraga ${ }^{39,40}$ that models a protein by a chain of connected beads characterized by dihedral angles and the van der Waals interactions among the beads. When parametrized suitably, the model can simulate protein secondary structures such as alpha helices, beta sheets, and loops. Here, our aim is to show that a targeted reweighting method can be designed that rapidly explores the (large) available configuration space of some degrees of freedom (loop regions) while maintaining more rigid structures (alpha helices) even in the modified trajectory.

The remainder of the work is organized as follows. In Sec. II, the targeted reweighting scheme is introduced along with our method of integration of the resulting equations of motion. In Sec. III, we analyze the double-well and Scheraga models with the targeted reweighting scheme, and in Sec. IV we summarize our results and the prospects for further development of the method.

\section{METHODOLOGY}

\section{A. Targeted reweighting scheme}

A targeted reweighting scheme is based on the identity for the Boltzmann average (with potential $V$ ) of any function $A\left(q^{N}\right)$

$$
\begin{aligned}
& \left\langle A\left(q^{N}\right)\right\rangle=\frac{\int d q^{N} A\left(q^{N}\right) e^{-\beta V\left(q^{N}\right)}}{\int d q^{N} e^{-\beta V\left(q^{N}\right)}} \\
& =\frac{\int d q^{N}\left\{A\left(q^{N}\right) e^{+\beta \Delta V\left(q^{N}\right)}\right\} e^{-\beta V^{*}\left(q^{N}\right)}}{\int d q^{N}\left\{e^{+\beta \Delta V\left(q^{N}\right)}\right\} e^{-\beta V^{*}\left(q^{N}\right)}} \\
& =\left\langle A\left(q^{N}\right) e^{+\beta \Delta V\left(q^{N}\right)}\right\rangle^{*} /\left\langle e^{+\beta \Delta V\left(q^{N}\right)}\right\rangle^{*} \\
& =\frac{\lim _{T \rightarrow \infty} \int_{t_{0}}^{t_{0}+T} A\left(q_{*}^{N}(s)\right) e^{+\beta \Delta V\left(q_{*}^{N}(s)\right)} d s}{\lim _{T \rightarrow \infty} \int_{t_{0}}^{t_{0}+T} e^{+\beta \Delta V\left(q_{*}^{N}(s)\right)} d s},
\end{aligned}
$$

where $V=(V+\Delta V)-\Delta V \equiv V^{*}-\Delta V$ defines $V^{*}$, a modified potential-energy surface upon which the dynamics is carried 
out, $\langle\cdots\rangle^{*}$ denotes an average with modified $\left(V^{*}\right)$ weighting, and $q_{*}^{N}$ denotes the modified trajectory. When generalized coordinates are used, the configuration-space integrals will involve the associated metric. ${ }^{11}$ For our purposes, we will assume that this metric is unity, as it can also be incorporated into a redefinition of the energy surface.

The last identity indicates that, with the assumption of ergodicity, the ensemble averages are to be evaluated as time averages over the trajectories generated from the modified surface. At each step of the dynamics on the modified surface, the data are reweighted with the factor $\exp (\beta \Delta V)$ to guarantee that the average is Boltzmann weighted. If barriers are reduced, the modified trajectory will explore the configuration space more rapidly. However, if the surface is distorted so much that $\Delta V \sim-V$, the modified trajectory will sample configuration space essentially uniformly. Then, as evident in the last equality, there will be many small contributions to the integrals in both numerator and denominator [the $\exp (\beta \Delta V)$ factor will be small] and few terms will contribute to the time integrals. Thus, a compromise on the degree of surface modification is required in order to optimize the numerical efficiency, and which terms in the potential are modified will be important factors to the success of the scheme.

\section{B. Equations of motion and method of integration}

To obtain the equilibrium distribution we introduce a dynamics according to the following generic Langevin equation: ${ }^{32,33}$

$$
\begin{aligned}
& d q_{j}(t) / d t=-\partial V\left(q_{1}, \ldots, q_{N}\right) / \partial q_{j}+\sqrt{2 D} \eta_{j}(t) \\
& (j=1,2, \ldots, N),
\end{aligned}
$$

where $D$ is a constant that will be fixed by association with the desired temperature, and $\eta_{j}(t)$ is a Gaussian, deltacorrelated, zero mean (white-noise) stochastic process whose correlation function is given by

$$
\left\langle\eta_{i}(t) \eta_{j}(s)\right\rangle=2 D \delta_{i j} \delta(t-s)
$$

The angular bracket denotes an average over realizations of the white noise. For convenience, all quantities in Eqs. (2.2) are written in dimensionless form. The Fokker-Planck equation that follows from this Langevin equation is 32,33

$$
\begin{aligned}
\frac{\partial P\left(q_{1}, \ldots, q_{N}, t\right)}{\partial t}= & \sum_{j=1}^{N} \frac{\partial}{\partial q_{j}}\left[\frac{\partial V\left(q_{1}, \ldots, q_{N}\right)}{\partial q_{j}} P\left(q_{1}, \ldots, q_{N}, t\right)\right] \\
& +D \sum_{j=1}^{N} \frac{\partial^{2}}{\partial q_{j}^{2}} P\left(q_{1}, \ldots, q_{N}, t\right)
\end{aligned}
$$

where $P\left(q_{1}, \ldots, q_{N}, t\right)$ is the joint probability distribution of the values of the generalized coordinates that is generated from the white-noise process. The unique time-independent solution of the Fokker-Planck equation is

$$
P_{\mathrm{eq}}\left(q_{1}, \ldots, q_{N}\right)=\lim _{t \rightarrow \infty} P\left(q_{1}, \ldots, q_{N}, t\right) \sim e^{-V\left(q_{1}, \ldots, q_{N}\right) / D} .
$$

The identification $D \equiv \beta^{-1}=k_{B} T$ then guarantees that the probability distribution constructed from the long-time solution of the Langevin equation will approach the Boltzmann distribution for temperature $T$. The solution will be obtained after a transient time that scales as $\sim e^{V_{b} / D}$, with $V_{b}$ the magnitude of the potential, as can be shown by a Kramers (Arrhenius)-based analysis. ${ }^{16}$

It is important to not confuse the above Langevin equation with "physical" Langevin equations that, for example, are used in position and velocity space to generate a model of a real, albeit approximate, dynamics, as used in Brownian dynamics simulations. ${ }^{31}$ In that situation, the actual dynamics of, for example, a fluid or a protein is being modeled and time correlation functions will describe the character of the relaxation to equilibrium. Here, no dynamical information is available or desired. The dynamics is used only as method to provide the correct Boltzmann distribution. A useful feature of this Langevin equation is that the noise term is not state dependent. That permits the use of an integration scheme that has great advantages, as now discussed.

Helfand $^{34}$ and Greenside and Helfand ${ }^{35}$ (see also the Appendix in Ref. 41) proposed procedures for numerically integrating stochastic differential equations. By the analogy with deterministic Runge-Kutta algorithms, they developed methods to estimate the value of a stochastic variable at time $t+h$ if its value at time $t$ is known. This is achieved by evaluating the right-hand side of the Langevin equation at selected points within each interval of length $h$, so that all moments of $x(t+h)-x(t)$ are given correctly to order $h^{k}$. The stochastic part is developed as a series in $h^{1 / 2}$, with the order of terms determined in probability. ${ }^{41}$ For the so-called $3{ }_{O}{ }_{S} 2_{G}$ algorithm used here, the order of the deterministic part is $k=4$, as in the nonstochastic fourth-order Runge-Kutta method and $k \sim 3.5$ for the stochastic part. The use of this high-order algorithm permits time steps that are large in comparison with more typical second-order integration methods popular for the integration of force-field-based equations of motion.

A useful check on the programing and the quality of the integration can be obtained for the nonstochastic version of Eq. (2.2a). If this equation is multiplied by $d q_{j}(t) / d t$ and summed up over $j$, it is straightforward to show that

$$
\sum_{j=1}^{N} F_{q_{j}}^{2}(t)=-d V / d t
$$

where the $F_{q_{j}}(t)$ are the forces on the $q_{j}$. Therefore, the total potential energy must monotonically decrease with time until a stationary point is reached, and Eq. (2.5) can be numerically verified from the integration data. 


\section{Potentials for the models}

\section{Double-well model}

A simple model for which the marginal (for each coordinate) equilibrium distributions are known analytically is based on a set of $N$ double-well potentials:

$$
V\left(q_{j}\right)=\left(1-q_{j}^{2}\right)^{2}+c_{j} q_{j} \quad(j=1, \ldots, N)
$$

with $c_{j}$ setting the scale of the asymmetry of the potential. For $c_{j}=0$, the two minima are located at \pm 1 and the maximum is $V_{b}=1$. For convenience, we will refer to these degrees of freedom as particles. The value of the barrier relative to the temperature $\beta V_{b}$ determines the rate at which the stochastic process can explore the configuration space, whose dimension scales as $2^{N}$. If the two states for each particle are viewed as two values of a dihedral coordinate for a chain molecule with $N$ dihedral angels, then a geometric realization of the $2^{N}$-dimensional state space can be obtained. Then, the possible states obtained from the solution of the Langevin dynamics can be visualized.

\section{Chain model}

This model is adopted from the work of $\mathrm{He}$ and Scheraga. ${ }^{40}$ It consists of a set of beads connected by bonds of fixed (unit) length and fixed angles, with dihedral angles that are rotatable according to a dihedral potential. In addition, the beads interact with a pairwise potential that prevents bead overlaps. The parametrization of the potential used here is somewhat different from that of He and Scheraga's because our goals are quite different. There are $n_{\mathrm{ob}}$ beads $\left(1,2, \ldots, n_{\mathrm{ob}}\right)$ with $n_{\mathrm{od}}=n_{\mathrm{ob}}-3$ dihedral angles $q_{j}$ $\left(j=1,2, \ldots, n_{\mathrm{od}}\right)$. The $q_{j}$ th dihedral angle is between beads $\mathbf{R}_{j+1}$ and $\mathbf{R}_{j+2}$. The bead positions can be generated by the recursive formula

$$
\begin{aligned}
\mathbf{R}_{i}-\mathbf{R}_{i-1}= & -\cos \theta\left[1+\cos \left(q_{i-3}\right)\right]\left(\mathbf{R}_{i-1}-\mathbf{R}_{i-2}\right) \\
& -\cos \left(q_{i-3}\right)\left(\mathbf{R}_{i-2}-\mathbf{R}_{i-3}\right)-\sin \left(q_{i-3}\right)\left[\left(\mathbf{R}_{i-1}\right.\right. \\
& \left.\left.-\mathbf{R}_{i-2}\right) \times\left(\mathbf{R}_{i-2}-\mathbf{R}_{i-3}\right)\right] \quad\left(4 \leqslant i \leqslant n_{\mathrm{ob}}\right)
\end{aligned}
$$

when given the positions of the first three beads. The angle $\theta$ is the (fixed) angle formed by any three successive beads and is set to the tetrahedral value, $\cos \theta=-1 / 3$. The positions of the first three beads are specified for convenience as

$$
\mathbf{R}_{0}=(\sin \theta, 0, \cos \theta), \quad \mathbf{R}_{1}=(0,0,0), \quad \mathbf{R}_{0}=(0,0,1) .
$$

The dihedral terms in the potential have the form

$$
\begin{aligned}
& V_{\mathrm{dih}}^{(1)}\left(q_{j}\right)=\varepsilon_{1}\left\{2-\frac{1}{8}\left[1-\cos \left(q_{j}+\phi\right)\right]^{4}\right\}, \\
& V_{\mathrm{dih}}^{(2)}\left(q_{j}\right)=\varepsilon_{2}\left[q_{j}^{2}-1\right]^{2} .
\end{aligned}
$$

The first dihedral potential $V_{\text {dih }}^{(1)}$ is adapted from $\mathrm{He}$ and Scheraga. ${ }^{40}$ It has a phase that can simulate different protein structures such as $\alpha$ helices and $\beta$ strands. We will set the phase $\phi$ to $\pi-1.4038$ (in radians) in some of the dihedral potentials to simulate $\alpha$-helical regions. The minimum of this potential is at $q_{i}=1.4038$, and this leads to a helix repeat of 3.6 beads, the protein repeat value. The second potential $V_{\mathrm{dih}}^{(2)}$ is used to model dihedrals that are strongly confined to certain angular regions from the presence of specific interactions in a protein environment. For example, in a loop region, there may be environmental factors that restrain the loop dihedrals to a discrete number of states. These will tend to have high barriers that we model with $V_{\text {dih }}^{(2)}$. Note that while it does not have a periodic form, it rises fast enough near $\pm \pi$ to not cause a problem.

The bead-bead interaction potential $V^{\mathrm{bb}}$ has the form

$$
V_{k l}^{\mathrm{bb}}\left(R_{k l}\right)=\varepsilon_{k l}\left[\left(\frac{\sigma_{k l}}{R_{k l}}\right)^{2 p_{k l}}-\tau_{k l}\left(\frac{\sigma_{k l}}{R_{k l}}\right)^{p_{k l}}\right] .
$$

Here, $\varepsilon_{k l}, \sigma_{k l}, \tau_{k l}$, and $p_{k l}$ are, respectively, energy, length, nature of potential (purely repulsive $\tau_{k l}=0$, or with an attractive well form $\tau_{k l}>0$ ), and steepness parameters for the potential. For a van der Waals interaction, $p_{k l}=6$. The presence of nonbonded interactions will naturally modify, for example, the $\alpha$-helical structure defined by the dihedral potential, $V_{\text {dih }}^{(1)}$.

In many atom-based force fields, the pure dihedral potentials have small barriers (relative to $k_{B} T$ ) and the actual dihedral barriers are determined by the " $1-4$ " interactions. That is, in the dihedral potential for beads labeled 1-2-3-4 where the dihedral angle corresponds to rotation about the axis defined by beads $2-3$, the nonbonded interaction between beads 1 and 4 provides the significant barrier to dihedral transitions. Such interactions are in part responsible for the preference for certain dihedral angles in proteins, as summarized in Ramachandran plots. ${ }^{42,43}$ Consequently, we construct effective dihedral potentials by adding to the dihedral potentials of Eqs. (2.8a) and (2.8b) the corresponding contributions from the 1-4 bead-bead interactions in Eq. (2.9).

The total potential between pair of beads from the nonbonded interaction

$$
V^{\mathrm{bb}}=\sum_{k=1}^{n_{\mathrm{od}}} \sum_{l=k+3}^{n_{\mathrm{ob}}} V_{k l}^{\mathrm{bb}}\left(R_{k l}\right)
$$

The derivative expression (for $j=1,2, \ldots, n_{\mathrm{od}}$ ) (Ref. 40)

$$
\frac{\partial \mathbf{R}_{i}}{\partial q_{j}}=\left(\mathbf{R}_{j+2}-\mathbf{R}_{j+1}\right) \times\left(\mathbf{R}_{i}-\mathbf{R}_{j+1}\right) \quad\left(j+3 \leqslant i \leqslant n_{\mathrm{ob}}\right)
$$

and

$$
\frac{\partial \mathbf{R}_{i}}{\partial q_{j}}=0 \quad(1 \leqslant i \leqslant j+2)
$$

and the use of the chain rule leads to the bead-bead contribution to the force on the $j$ th dihedral in the form

$$
\begin{aligned}
\mathbf{F}_{j}= & -\sum_{k=1}^{j+2} \sum_{\substack{l=k+3 \\
l \geqslant j+3}}^{n_{\mathrm{ob}}} \frac{\partial V_{k l}^{\mathrm{bb}}\left(R_{k l}\right)}{\partial R_{k l}} \frac{\mathbf{R}_{k l}}{R_{k l}} \cdot\left[\left(\mathbf{R}_{j+2}-\mathbf{R}_{j+1}\right)\right. \\
& \left.\times\left(\mathbf{R}_{l}-\mathbf{R}_{j+1}\right)\right] .
\end{aligned}
$$

The expression for the force on the $j$ th dihedral from isolating the 1-4 bead-bead interactions on the $j$ th dihedral is 


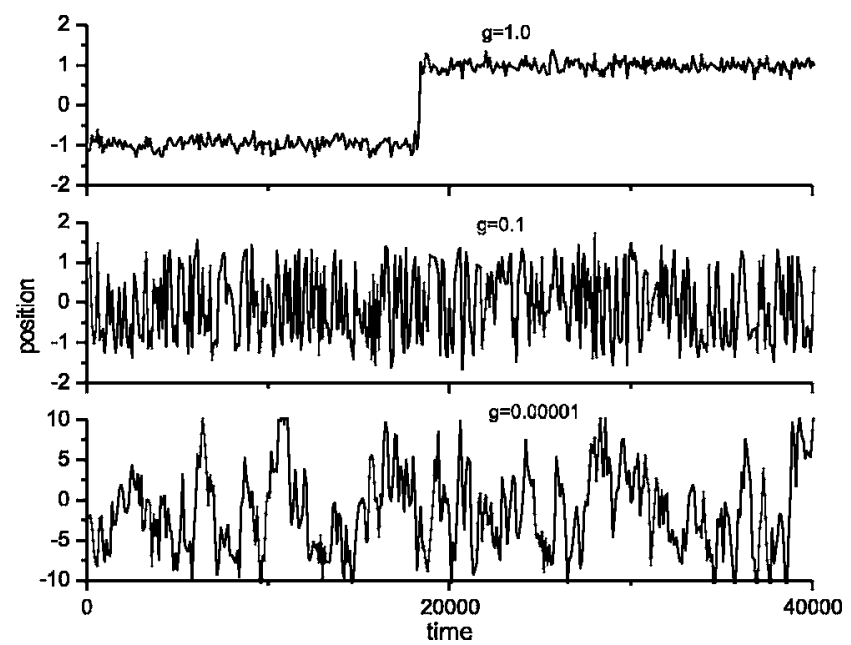

FIG. 1. The first 40000 time units of the trajectories for a symmetric double-well potential with temperature $D=0.1$ for $g=1.0,0.1$, and 0.00001 . The $g=1.0$ trajectory has transitions on a scale of 10000 time units and cannot predict the equilibrium distribution even for a 100000 time unit run. The $g=0.1$ trajectory exhibits numerous transitions between the two states around positions \pm 1.0 . For $g=0.00001$, the scaled surface has been flattened so that a much larger space than indicated by the true potential is explored, leading to fewer excursions between the two states than for $g=0.1$. For all the figures, the temperature, energy, coordinates, and time are given in dimensionless units.

$$
\begin{aligned}
\mathbf{F}_{j}^{(14)}= & -\sum_{k=j}^{j+2} \frac{\partial V_{k, k+3}^{\mathrm{bb}}\left(R_{k, k+3}\right)}{\partial R_{k, k+3}} \frac{\mathbf{R}_{k, k+3}}{R_{k, k+3}} \cdot\left[\left(\mathbf{R}_{j+2}-\mathbf{R}_{j+1}\right)\right. \\
& \left.\times\left(\mathbf{R}_{k+3}-\mathbf{R}_{j+1}\right)\right] .
\end{aligned}
$$

The sum of this force and the dihedral forces defines the effective force $F_{j}^{\text {eff }}$ on the $j$ th dihedral:

$$
\mathbf{F}_{j}^{\mathrm{eff}}=-\frac{\partial\left(V_{\mathrm{dih}}^{(1)}\left(q_{j}\right)+V_{\mathrm{dih}}^{(2)}\left(q_{j}\right)\right)}{\partial q_{j}}+\mathbf{F}_{j}^{(14)} .
$$

Given a set of dihedral angle values, $q_{j}\left(j=1, \ldots, n_{\text {od }}\right)$, the bead positions are available from Eqs. (2.7a) and (2.7b) the corresponding potentials from Eqs. (2.8a)-(2.10)and forces from Eqs. (2.12)-(2.14). These forces are used in the equation of motion in Eqs. (2.2a) and (2.2b) to provide the information for the stochastic integrator.

\section{RESULTS}

\section{A. Double-well models}

The symmetric, single double-well potential of Eq. (2.6), with its barrier $V_{b}=1.0$, provides a simple test of the reweighting scheme. In all the following, the simplest form of potential modification, $V^{*}=g V$ with the scale factor $g$ satisfying $0<g \leqslant 1.0$, is used. In Fig. 1 the trajectories of the coordinate $q$ are given for $g=1.0,0.1$, and 0.00001 and a temperature of $D=0.1$. There are 20 integration steps $(h=0.05)$ for each unit of time. Only the first 40000 out of 100000 time units are shown. The $g=1.0$ trajectory has transitions on a scale of 10000 time units in accord with the Kramers (Arrhenius) rate theory expression ${ }^{16}$ $k=\sqrt{\omega_{0} \omega_{b}} / 2 \pi e^{-V_{b} / D}=4 \sqrt{2} / \pi e^{-V_{b} / D} \sim 0.0000817$, where $\omega_{0}$ and $\omega_{b}$ are the well and barrier frequencies, respectively, and the second equality is appropriate to the double-well poten-

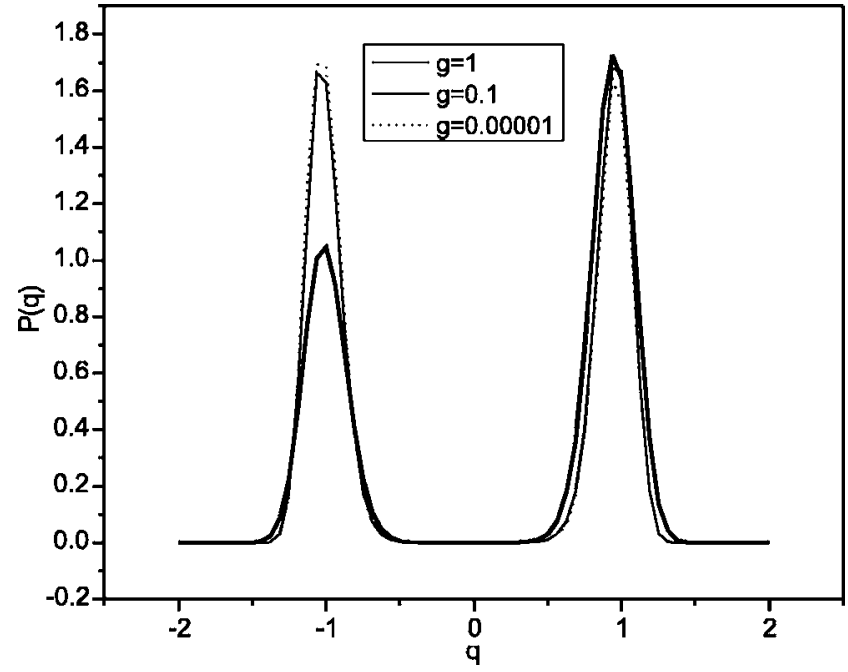

FIG. 2. Histograms for a symmetric double-well potential with temperature $D=0.1$ for $g=1.0,0.1$, and 0.00001 . The $g=1.0$ result cannot be accurate for the time range of the data. The $g=0.1$ and 0.00001 histograms are numerically coincident with the analytic $P_{\text {eq }}(q)$ expression.

tial. The $g=0.1$ trajectory has numerous jumps between the two minima of the potential located at \pm 1.0 , and the position remains within this range. The $g=0.00001$ trajectory shows a consequence of too strong a scaling; the potential $g V$ has been flattened to the extent that the particle explores a larger space than is desired and, consequently, the number of times it samples the important regions around \pm 1.0 is greatly reduced relative to, for example, the $g=0.1$ trajectory. Thus, for a given time interval, as $g$ is decreased from unity, where there are insufficient number of transitions, to a small value, where the exploration of space is too large and not sufficiently concentrated in the important regions (around the minima of the potential surface) there should be a span of $g$ values that are computationally efficient. Qualitatively, it is clear that values of $g$ such that $g V_{b} / D \sim 1$ are appropriate. If this factor is somewhat bigger than 1 , the sampling will produce many transitions when the time scale is chosen as the Kramers relaxation time (many transitions on this time scale) while still mainly sampling from the regions of low potential.

In the current example with $V_{b}=1.0$ and $D=0.1$ a value of $g \sim 0.1$ should be optimal. It is clear that too large a value will not be accurate. However, for this one-dimensional case, the computational efficiency does not drop off even for quite small values of $g$ such as $g=0.00001$. That is most readily seen in the histograms corresponding to the equilibrium probabilities displayed in Fig. 2. The $g=1.0$ result is inaccurate (and actually useless since it will depend completely on how much data are used) while both the $g=0.1$ and 0.00001 results are, to the precision inherent to histograms, exactly what the analytic $P_{\text {eq }}(q)$ answer predicts. It should be noted that the double-well potential rises so steeply away from the minima that the flattening of the potential is not as great as it would be for other potential forms. This will slow the increase in the sampling interval and mitigate the increase in configuration-space exploration as $g$ decreases.

To examine the effect of multiple degrees of freedom on the quality of the reweighting scheme, a six-dimensional ver- 


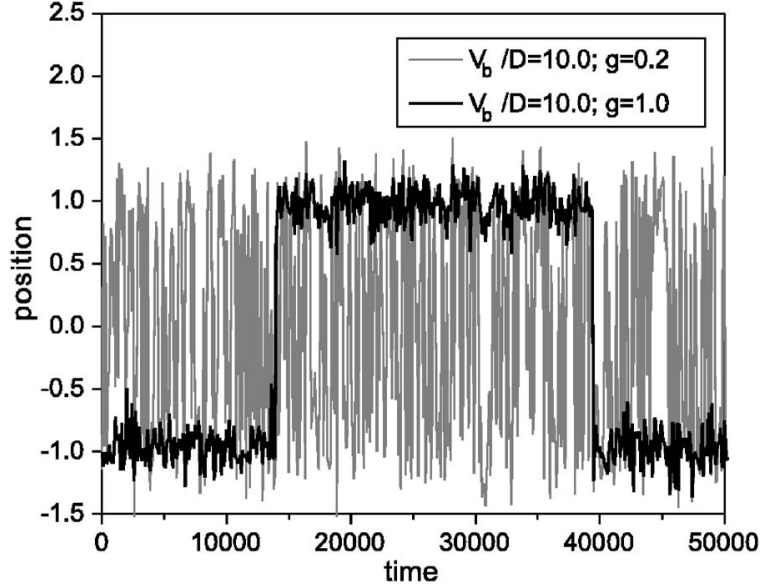

FIG. 3. Trajectories for the first particle in the six-particle symmetric double-well simulation with temperature $D=0.1$ for $g=1.0$ and $g=0.2$ are displayed over 50000 steps. The rest of the data are similar.

sion of the symmetric double-well model was investigated, with the barriers $V_{b}=1$ for all the wells. There are then $2^{6}=64$ states in the model, where a state is labeled by converting to decimal representation the string of six binary digits that specify whether each particle is to the "left" or "right" of each individual barrier, at a particular time. The particles are all started in the left wells, with positions -1.0. The results presented below on the equilibrium properties are insensitive to the initial conditions. The temperature parameter is set to $D=0.1$ and, for the modified trajectory runs, $g=0.2$; thus, $V_{b} / D=10.0$ and $g V_{b} / D=2.0 .8 \times 10^{6}$ steps were run for $g=1$ and $0.8 \times 10^{6}$ for $g=0.2$. For this scale factor, the efficiency should be close to optimal as discussed above. A scale factor of $g=0.01$ was also used to explore the optimization issue in this six-dimensional case. The Langevin equation is integrated with a step size $h=0.01$.

The $i$ th singlet, marginal probability obtained from

$$
P\left(q_{i}\right)=\int d q_{1}, \ldots, d q_{i-1} d q_{i+1}, \ldots, d q_{N} P\left(q_{1}, q_{2}, \ldots, q_{N}\right)
$$

is the same as the one-dimensional probability for the $i$ th degree of freedom because the potential is separable. Note that the integrator does not reduce the system to independent equations when the reweighting method is used, as is evident in the reweighted trajectory expression in the last equality in Eq. (2.1).

The trajectory over 50000 time units is displayed in Fig. 3 for one particle. The rest of the data are similar in character. The $g=1.0$ trajectory shows transitions on a scale of 10000 time units in accord with the Kramers' estimate (whose accuracy requires a large barrier relative to the temperature). A histogram (not shown) over the entire $g=0.2$ reweighted data shows that the sampling of the double well is mainly concentrated in the well regions. The singlet probabilities, every 100000 time units, of being on the left side for the interval from 200000 to 800000 time units are displayed in Fig. 4. They show that the $g=1$ results do not converge to the correct value of 0.5 over this interval, while the $g=0.2$ results certainly do. (There is some imprecision in the definition of probability of being in either well, of


FIG. 4. Probabilities for each particle to be in the left well for $g=1.0,0.2$, and 0.01 from 200000 to 800000 time units for the six-particle symmetric double-well simulation with temperature $D=0.1$. The $g=1.0$ results are clearly not converged but will do so when the data over 8000000 steps are used. The results with $g=0.2$ are essentially converged by 100000 time units, while the $g=0.01$ results are not converged over the 800000 time unit interval.

course.) The problem with "excess" scaling is evident in the bottom panel of Fig. 4. The use of $g=0.01$ degrades the quality of the results for a given integration time. The reweight factor is a sum of the various contributions to the total potential. Thus, as the number of reweighted degrees of freedom increases, the likelihood of obtaining, for $g \ll 1$, a small value of $\exp (\beta \Delta V)=\exp (\beta(g-1) V) \sim \exp (-\beta V)$ increases, and the number of terms contributing to the time integrals in Eq. (2.1) decreases, compromising their accuracy for a given integration time.

The singlet probabilities are integrals over the remaining particle coordinates and, as such, decrease the information content. A more severe measure can be found in the state space distributions; that is, how often each state is visited over the trajectory. Figure 5 presents the fraction of time each of the 64 states is visited over the simulation time. It should be clear that, even though the sampling is ten times longer for $g=1$, the coverage of state space is much closer to uniform for the $g=0.2$ data. Ideally, the fractional coverage should be $1 / 64=0.015625$. Since we use a definition of state that, for a given particle, excludes the interval $(-0.1,+0.1)$ around the barrier, there is a slight counting error.

An asymmetric double well with $c_{j}=-0.2$ for all $j$ was run. The system was started with all particle coordinates at -1.0 , and the surfaces are all downhill in the "forward" direction toward the particle coordinates +1.0 . The temperature is $D=0.075$ and the scale factor is set as $g=0.2$. The barriers in the forward (reverse) directions are 0.8 (1.2). Again, 8 


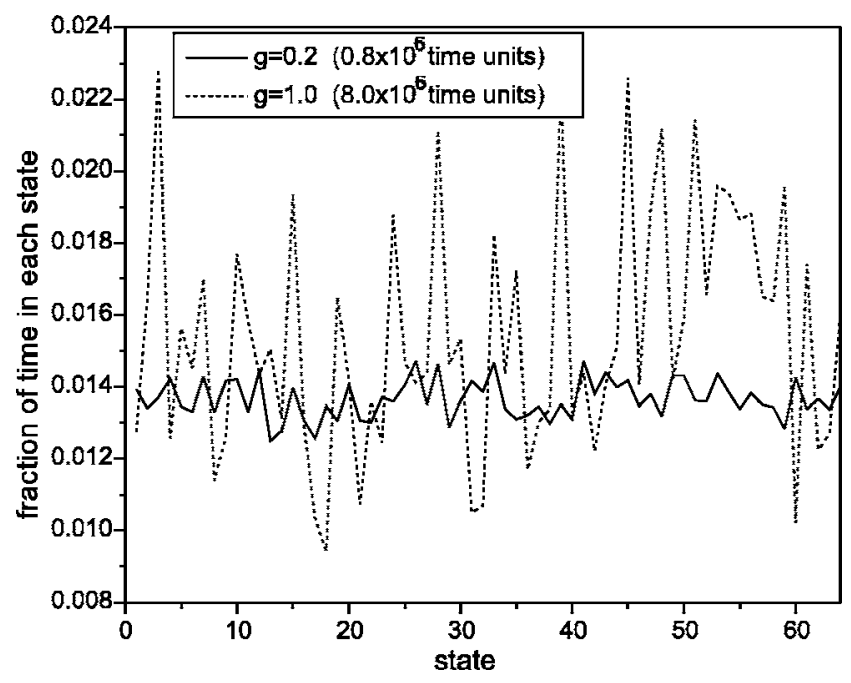

FIG. 5. The fractions of time spent in each of the possible 64 states for $g=1.0$ (over 8000000 time units) and 0.2 (over 800000 time units) for the six-particle symmetric double-well simulation with temperature $D=0.1$. The sampling for $g=0.2$ is much more uniform than for $g=1.0$ even though there are ten times more data for the latter than the former.

$\times 10^{6}\left(0.8 \times 10^{6}\right)$ steps were run for the $g=1.0(g=0.2)$ simulations. Figure 6 shows two of the particle trajectories for $g=1.0$ over 100000 steps. After their transitions from -1.0 to +1.0 , both stay in the final state $($ all +1.0$)$ for almost all the remaining time, with one or two quick excursions back to -1.0 . These results show that even though the particles become trapped in the low-energy regions, the singlet probability of being in the low-energy state of 0.994779 cannot be properly predicted because there are so few transitions over this long interval. The modified trajectory data exhibit many transitions. The probabilities cannot be read off these plots since only after reweighting will the distribution be correctly Boltzmann. The plot does indicate that there are many transitions and therefore must lead to the correct results. The singlet probabilities are between 0.994 and 0.995 for all six particles when the data for 100000 and longer times are tabulated. Examination of the fraction of time spent in the 64 states shows that the distribution among states of equivalent energy (for example, the six states where one particle is around -1.0 and the other five are around +1.0 ) is quite uniform.

So far, all the barriers have been assumed to be of the same height. To investigate targeted reweighting, there need to be barriers of different heights. Using the same six-particle model, the first three barriers are set to $V_{b}=1$ and the other three are set to $V_{b}=3$. For the temperature $D=0.1$ and a scaling factor $g=0.3$, there should be many transitions for the $V_{b}=1$ barriers but very few for the $V_{b}=3$ barriers over the $0.8 \times 10^{6}$ time units of the modified trajectory. Trajectories were generated for (1) no scaling, (2) scaling with $g=0.3$ for the first three and $g=0.1$ for the other three particles, and, as a check, (3) scaling the first three with $g=0.3$ and $g=1.0$ for the other three particle potentials. The targeted reweighting should provide the equilibrium probabilities for all six coordinates and it does. Scaling only the first three particle potentials should equilibrate them but fail to cause transitions for the other three, and this is the result. Of course, if it were

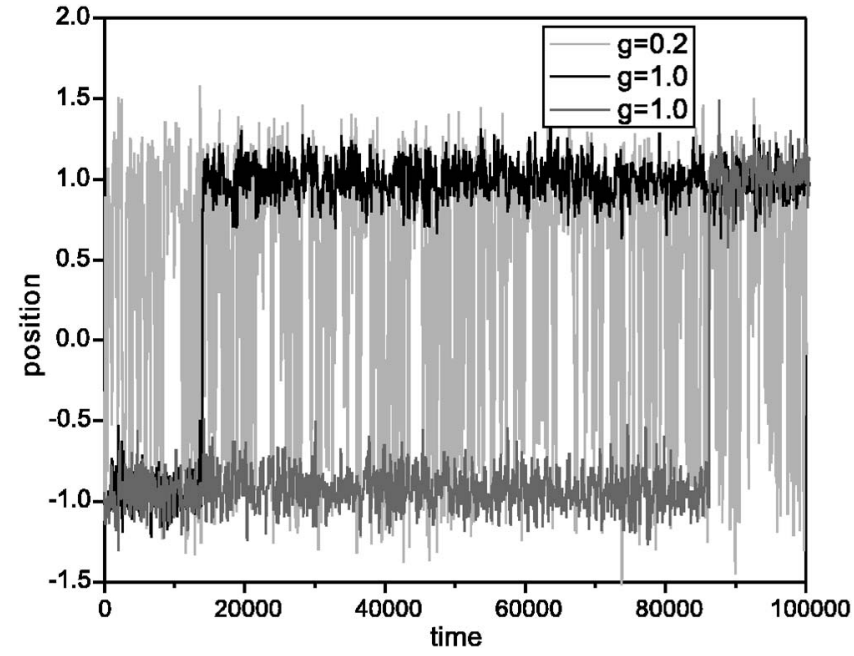

FIG. 6. Trajectories for $g=1.0$ of two of the six particles in the asymmetric double-well simulation with temperature $D=0.075$ over 100000 time units. There are just a few transitions in the remaining data (8 000000 time units) because the particles become trapped in the lower-energy state. The modified trajectory with $g=0.2$ exhibits numerous transitions over this interval and provides accurate probabilities.

possible to run the trajectory for $\sim e^{V_{b} / D}=e^{30}$ time units, the true equilibrium distribution would be obtained. Analogous simulations were run where there is asymmetry $c_{j}=-0.1$ for all $j$ in order to lead to a final state that is energetically distinguishable from the other states.

Instead of plotting these trajectory-based data in various ways, as done in the previous examples, we illustrate the results by mapping the 64 states onto the geometry of the protein model discussed in Sec. II. At each step, the particles' locations on either the left or right side of the barrier position are assigned, respectively, to the tetrahedral $(-1)$ or $\alpha$-helical $(+1)$ conformers. The all-tetrahedral configuration $(-1,-1,-1,-1,-1,-1)$ is arbitrarily selected as the initial state. The all $\alpha$-helical configuration is defined as $(+1,+1$, $+1,+1,+1,+1)$ for all six particles and is the low-energy, "native" state, for the asymmetric runs. Three particles, 0, 1, and 2 , are needed to define the origin of the chain and, therefore, there are a total of nine particles (beads) specified. The data for the states visited are shown in Fig. 7 for the three cases of no scaling and uniform and targeted scaling. The top panel shows that the chain stays in its initial state, the middle shows that $2^{3}=8$ states are sampled, and the bottom that all $2^{6}=64$ are visited, including, of course, the native state displayed as black. Thus, targeted reweighting has the desired property of distinguishing barriers of varying heights. It accelerates the exploration of configuration space for the desired degrees of freedom and is computationally efficient by not increasing the exploration of the high-barrier degrees of freedom.

\section{B. Chain model}

A feature of proteins is their use of combinations of secondary structure elements to form motifs. ${ }^{42}$ One of the simplest motifs is the helix-turn-helix (HTH) consisting of two $\alpha$ helices that are connected by a loop region. ${ }^{42,44}$ In DNA binding by proteins involved in transcriptional regulation, 


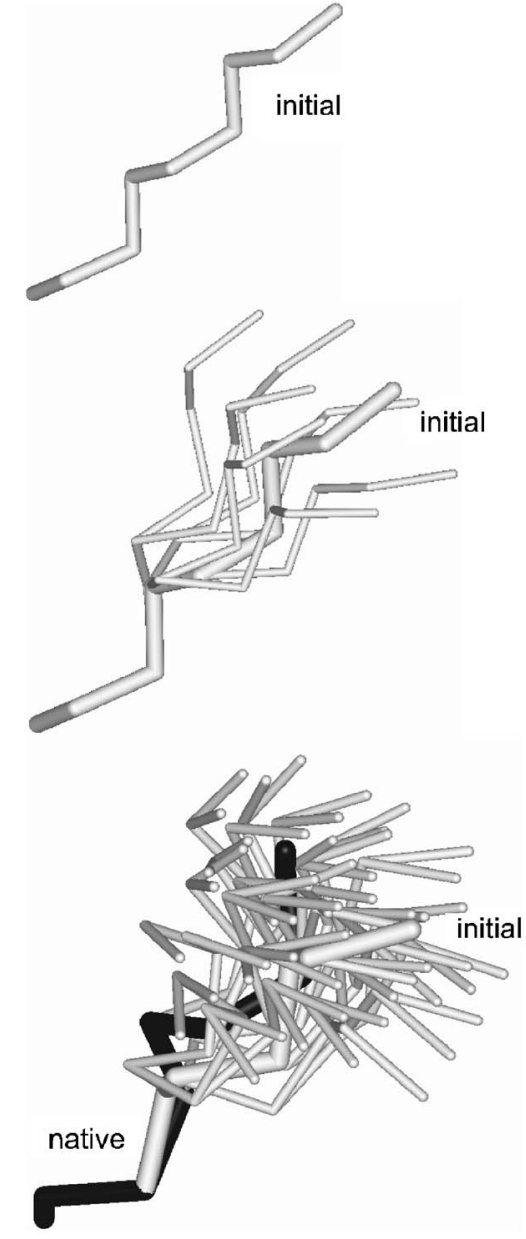

FIG. 7. Top: Extended initial state using Boltzmann sampling. The system remains in the initial state. Middle: Global scaling (the total potential is modified) with only sufficient scaling to overcome the first set of three low barrier dihedrals. Bottom: Targeted reweighting that can overcome all six dihedral barriers. The black line is the native state, where all angles correspond to the $\alpha$-helical dihedrals.

the HTH motif is extensively used. ${ }^{45}$ The loop region in this motif usually has four residues and the helical regions are a mix of hydrophobic and hydrophilic residues. ${ }^{44}$ The motif is defined not only by the HTH residues but also by the DNA and protein milieu. The issue here is if targeted reweighting can be used to generate HTH-like structures. That is, can geometries with two helices separated by stable loop-based structures be generated starting from an all-helical conformation? Our version of the He and Scheraga ${ }^{40}$ model introduced in Sec. II provides a potential function with dihedrals of two characters: $V_{\text {dih }}^{(1)}$ that simulates $\alpha$-helical structures and $V_{\text {dih }}^{(2)}$ that, with its double-well character, can produce two distinct states for each such degree of freedom. The bead-bead interaction term $V^{\mathrm{bb}}$ [Eq. (2.10)] prevents chain overlap and further restricts and modifies the configuration-space probabilities.

The chain has 29 dihedrals of which 1-13 have $\alpha$-helical potentials $V_{\text {dih }}^{(1)}$ with $\varepsilon_{1}=3$ and $\sigma=1.2,14-17$ have $V_{\text {dih }}^{(1)}$ of loop character $\left(\varepsilon_{1}=0\right)$ and $V_{\text {dih }}^{(2)}$ potentials with barrier $\varepsilon_{1}=2$, and 18-29 have $\alpha$-helical potentials $V_{\text {dih }}^{(1)}$ of with $\varepsilon_{1}=3$ and $\sigma=1.2$. For $\varepsilon_{1}=3$ in $V_{\mathrm{dih}}^{(1)}$ there is a single minimum around 1.4038 (radians) and its maximum value is $6 \mathrm{u}$, providing a

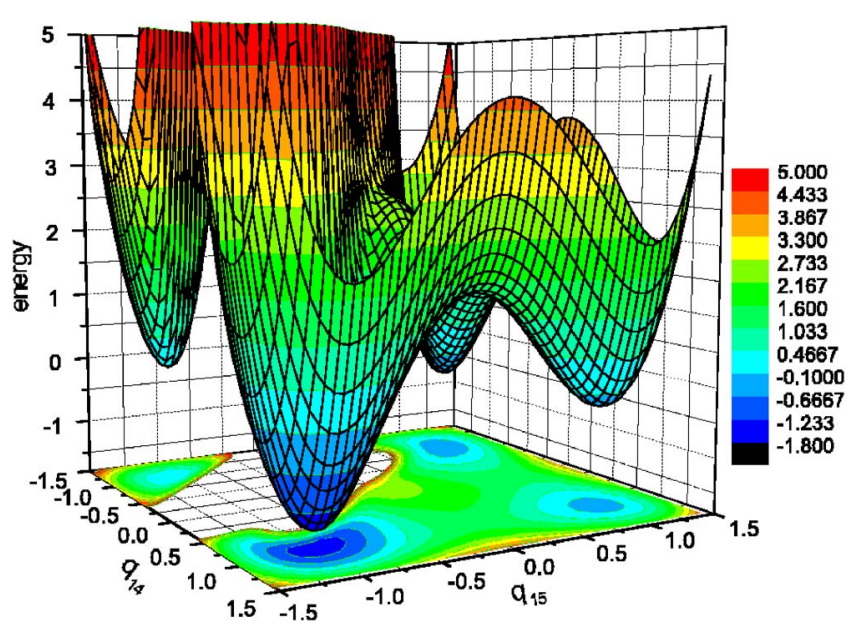

FIG. 8. The potential surface for loop dihedrals $q_{14}$ and $q_{15}$ with all other dihedrals fixed at their $\alpha$-helical values. The surface is truncated at high energy for purposes of display. There are four minima with the lowest at $q_{14} \sim 1.0$ and $q_{15} \sim-1.0$. That the minima are not equivalent, and the presence of the large barriers arises from the influence of the bead-bead interactions.

confining potential that is three times larger than the doublewell barrier of $2 \mathrm{u}$ in $V_{\mathrm{dih}}^{(2)}$. There are five different bead types with pairwise interactions that can either be repulsive or have a minimum. The bead assignment we use corresponds to the $\mathrm{He}$ and Scheraga ${ }^{40} \alpha$-helical chain [see their Eq. (4.2)]. The temperature will be set low enough that, in principle, there should be little tendency to destroy the $\alpha$-helical segments or go over the loop segment barriers. However, the bead-bead interaction produces an exceedingly complex potential surface in the dihedral space and a highly coupled dynamics, as is evident from the force expression in Eq. (2.12). Furthermore, the difference in character of the dihedral and the bead-bead potentials leads to the difficulty of quite different time scales. On the repulsive branch of the bead-bead interaction the forces get large necessitating a small $h$ while, in order to surmount the dihedral barriers, as large as possible a step size is desired. If only mechanics were required, integrating the equations of motion in Eqs. (2.2a) and (2.2b) would be straightforward since the system would just go to its minimum-energy state based on the initial conditions, as shown in Eq. (2.5), and the repulsive branch of the potential would not be accessed, assuming an appropriate initial condition. However, with the random force term that serves to sample from a Boltzmann distribution, it is possible to generate configurations where beads are relatively close and produce large forces. These considerations in part indicate the importance of using the targeted reweighting method. The terms in the potential for $\alpha$ helices should not be reweighted while those for the loop region should.

As an example that illustrates the complexity of the potential surface consider two loop dihedrals, 14 and 15, and the remaining 27 dihedrals as $\alpha$ helical. Because the surface is 29 dimensional, we keep dihedrals $1-13$ and 16-29 at their $\alpha$-helical values and rotate about dihedrals $q_{14}$ and $q_{15}$, as shown in Fig. 8. There are minima of different energies at approximately $(1,-1),(-1,1),(1,1)$, and $(-1,-1)$ in order of increasing energy. That the minima are unequal is a conse- 

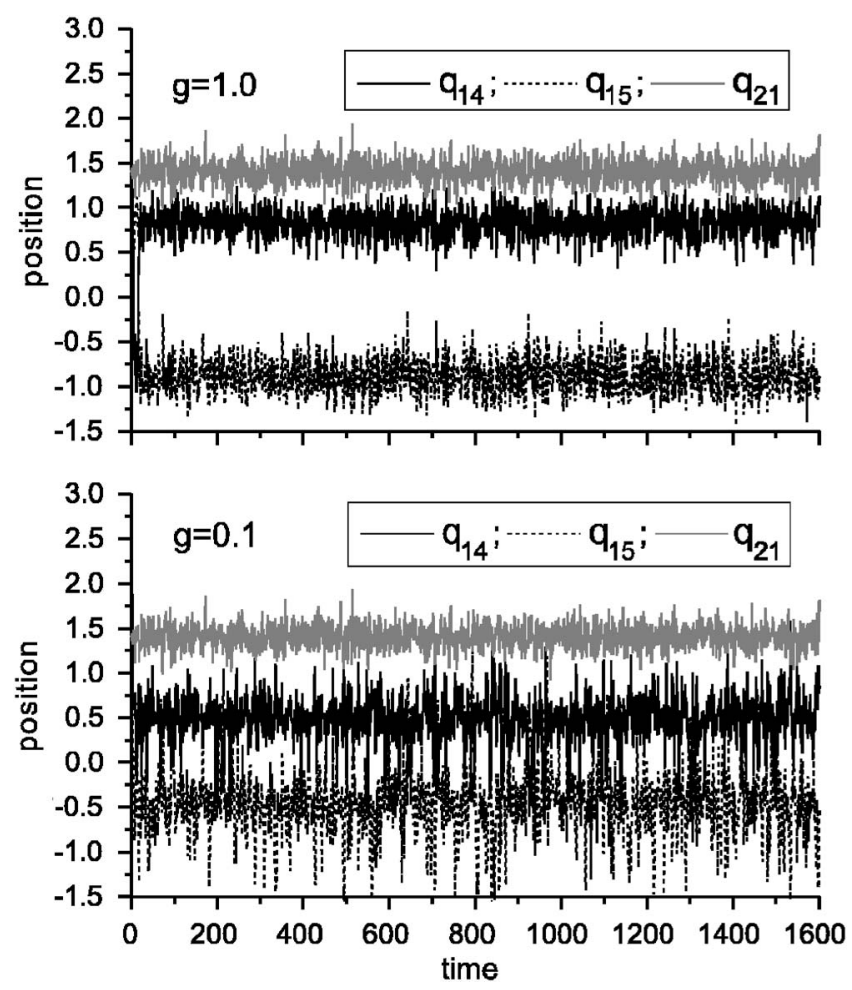

FIG. 9. Trajectories of $q_{14}, q_{15}$, and $q_{21}$ for the case of two loop dihedrals $\left(q_{14}\right.$ and $q_{15}$ ) and all others $\alpha$ helical, with $D=0.2$ for $g=1.0$ and $g=0.1$. The trajectories of the remaining $\alpha$-helical dihedrals are similar to the $q_{21}$ trajectory. The $g=0.1$ trajectory shows transitions between conformations that are not present in the $g=1.0$ trajectory.

quence of the bead-bead interactions, as are the very large barriers (not evident in the figure since the larger values are cut off) connecting some of the minima. Indeed, the paths between the stable states are quite constricted from beads coming close together as $q_{14}$ and $q_{15}$ are changed. Of course, the true path is in the 29-dimensional space and it may well be that the oscillations in those coordinates about their $\alpha$-helical minima provide lower-energy paths for loop transitions.

The initial condition is chosen as all $\alpha$ helical $\left(q_{j} \sim 1.4\right.$, $j=1-29)$ and the equations of motion integrated with a small step size $h=0.001$ in order to protect against bead-bead clashes. The temperature $D=0.2$ and $g=1.0,0.1$, and 0.05 scaling values were used. Examination of the total potential energy at each step shows that the energy never gets very large. Experience showed that if the integrator's accuracy is lost, it does so catastrophically, with the energy increasing to very large values.

The trajectories of dihedrals $q_{14}, q_{15}$, and $q_{21}$ for $g=1.0$ and $g=0.1$ are displayed in Fig. 9. The $q_{21}$ trajectory shows that this dihedral, as do all the other $\alpha$-helical ones, stays about their initial values in both $g=1.0$ and $g=0.1$ trajectories. The trajectory for the loop dihedrals quickly reaches the minimum potential region, $(1,-1)$. However, once there, no more transitions occur indicating that the $g=1.0$ trajectory cannot sample the surface properly. In contrast, the $g=0.1$ and $g=0.05$ (not shown) trajectories do repeatedly sample a wide range of dihedral angles for $q_{14}$ and $q_{15}$, while, with the targeted reweighting scheme, the $\alpha$-helical dihedrals fluctu-

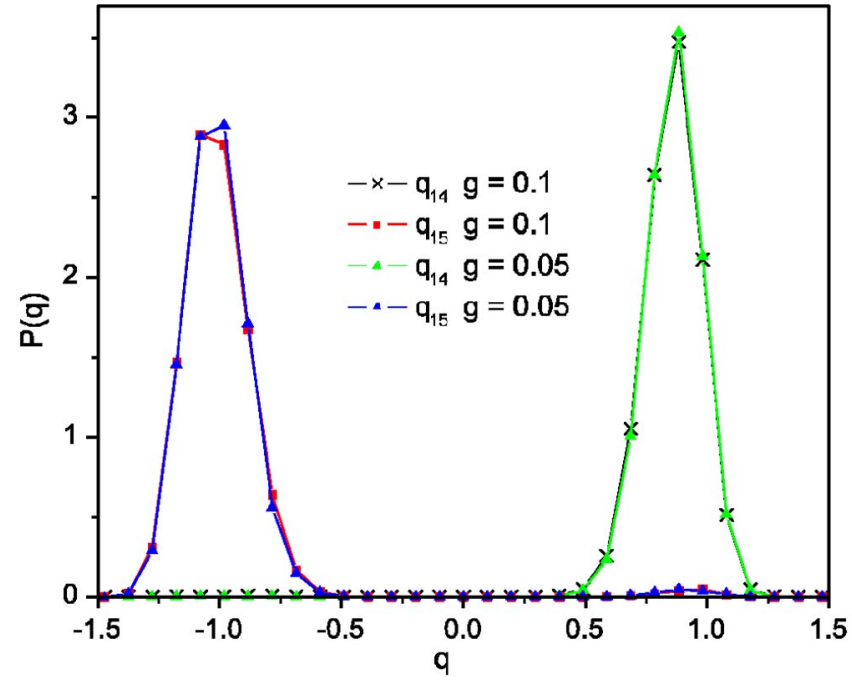

FIG. 10. Histograms of the positions of dihedrals $q_{14}$ and $q_{15}$ with $D=0.2$ for $g=0.1$ and $g=0.05$. The small peak where $q_{15} \sim 1.0$ shows that the targeted reweighting method can access the state around $q_{14} \sim 1.0$ and $q_{15} \sim 1.0$ that is higher in energy than the most populated state, where $q_{14} \sim 1.0$ and $q_{15} \sim-1.0$. The data for $g=0.1$ and $g=0.05$ are almost indistinguishable, indicating the accuracy of the method.

ate essentially like the $g=1.0$ trajectory. This is certainly not guaranteed, because the fluctuations in $q_{14}$ and $q_{15}$ are coupled to the other dihedrals through the bead-bead interactions.

The rapid approach to the minimum energy around $(1,-1)$ is not particularly sensitive to the initial conditions. Indeed, starting $q_{14}$ and $q_{15}$ around $(-1,1)$ or $(-1,-1)$ leads to similar results. The two-dimensional surface in Fig. 8 apparently does not give a good account of the true multidimensional surface; the fluctuations in the $\alpha$-helical degrees of freedom that are coupled via the bead-bead interactions to the loop dihedrals must provide a path for transition between the $(-1,-1)$ and $(1,-1)$ states. Note that if the temperature is set to zero, so that the dynamics is just mechanics, a trajectory started around $(-1,-1)$ rapidly approaches the coordinates appropriate to the bottom of that well.

Histograms of $q_{14}$ and $q_{15}$ are shown in Fig. 10 for $g=0.1$ and $g=0.05$. They show a small but definite population of the $(1,1)$ state that apparently is accessible with this scaling. Note that the histograms for $g=0.1$ and $g=0.05$ are almost the same, demonstrating the accuracy of the method. Since the $(1,-1)$ and $(1,1)$ states are separated in energy by approximately 1 , and the temperature is 0.2 , the relative population should be $\sim e^{-5} \sim 0.0067$, if the true equilibrium state would correspond to the other coordinates being maintained at their $\alpha$-helical minima. The relative population obtained by integration in Fig. 10 is $\sim 0.014$.

The situation with four loop dihedrals $q_{14}, q_{15}, q_{16}$, and $q_{17}$, and the other dihedrals $\alpha$ helical, was investigated, because this is evocative of a typical HTH motif. ${ }^{45}$ There is the possibility of sampling from $2^{4}=16$ configurations, based on the $V_{\text {dih }}^{(2)}$ dihedral potential, but the bead-bead interactions may constrain these possibilities. The temperature $D=0.15$ was used and the scale factors are $g=1.0$ and 0.4 . Based on the loop barrier of 2.0, there should be very few transitions with the $g=1.0$ trajectory, starting from the all $\alpha$-helical con- 

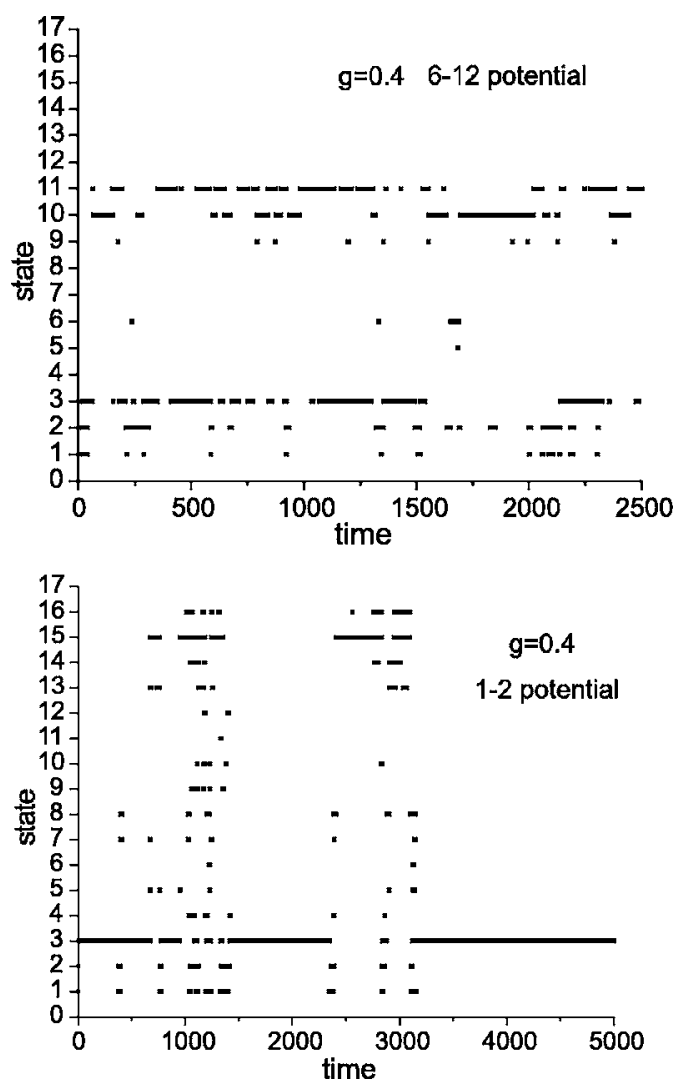

FIG. 11. The states visited by the modified trajectories for four loop dihedrals $\left(q_{14}, q_{15}, q_{16}\right.$, and $\left.q_{17}\right)$, with $D=0.15$ for $g=0.4$ for the 6-12 and 1-2 bead-bead potentials. Based on the dihedral potentials, there should be 16 states that could be populated. The bead-bead interactions remove the energy degeneracy of these states and the probabilities are nonuniform.

figuration. Trajectories were run for the bead-bead scale parameter, $p_{k l}$ [see Eq. (2.9)] set to either 6 or 1. The former generates the conventional 6-12 Lennard-Jones potential while the latter corresponds to a 1-2 potential, which is the most slowly varying of this class of potentials. We used the 1-2 potential to see if it could mitigate the problems that can arise from the mismatch of time scales between the dihedral and repulsive parts of the bead-bead potentials. The use of the 1-2 potential does permit the use of larger time step and these results are generated with $h=0.01$ versus $h=0.001$ for the $6-12$ potential.

The $g=1.0$ loop dihedral trajectories for both potentials show just a few transitions (2-4, depending on the dihedral) and only four states are visited during the simulation. When the scaling is set to $g=0.4$, there are many more transitions for the loop dihedrals relative to $g=1.0$, and the nonloop dihedrals still just fluctuate about their $\alpha$-helical values, as desired. A plot of the states visited is shown in Fig. 11. Out of the 16 possible states, 8 are visited using the $6-12$ potential and all 16 are visited using the 1-2 potential. In both simulations, state 3 , which corresponds to dihedral 15 having flipped from its initial value with the others remaining around their initial values, is the majority state, especially for the 1-2 potential. Note that this state corresponds to the minimum-energy state in the two-loop-dihedral case discussed above. For the 6-12 potential, there are substantial populations of stats 10 and 11 that correspond, respectively,
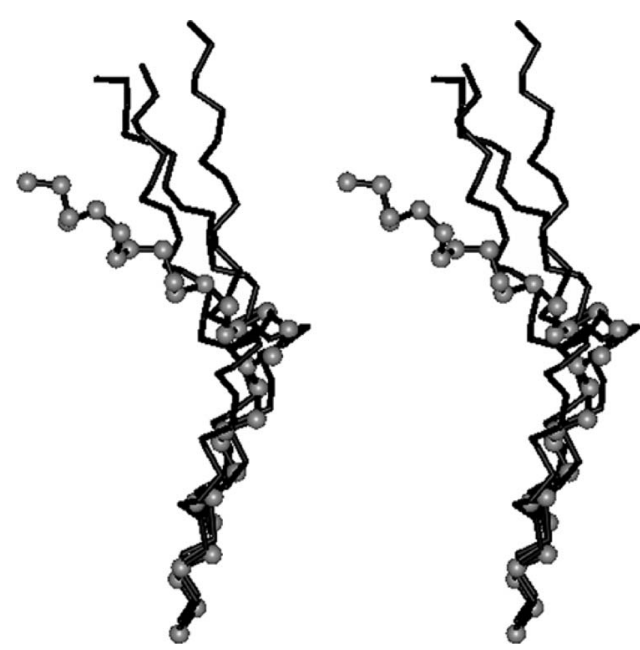

FIG. 12. Stereoview of the four most probable distinct conformers generated during the modified trajectories for four loop dihedrals $\left(q_{14}, q_{15}, q_{16}\right.$, and $q_{17}$ ), with $g=0.4$ for the $6-12$ bead-bead potential. State 3 , which is the most populated one (see Fig. 11), is displayed with ball and stick.

to dihedrals 14 and 17 and 15 and 17 flipped. The geometries of the states corresponding to $3,10,11$, and 2 are displayed in Fig. 12, for the 6-12 potential. It is evident that, relative to the all $\alpha$-helical state, these geometries are bent into forms that are similar to HTH structures.

\section{SUMMARY AND CONCLUDING REMARKS}

The time scale restrictions of atomistic, force-field-based simulations limit their usefulness for making predictions regarding structure in complex systems, such as proteins. If stable structures are to exist, there must be substantial barriers relative to the temperature. To integrate properly the equations of motion requires a small time step, since forces can become large, while to sample from a Boltzmann distribution in the presence of substantial barriers requires many steps. Often, in reduced descriptions used for folding studies, barriers are parametrized to be small relative to the temperature, since if the native state is known, one may parametrize the potential to guarantee approach to this state. If, however, attention focuses on using "accurate" atomistic force fields, then the problem of barriers in the potential surface cannot be avoided. In this work, we have explored a targeted reweighting method to speed up the exploration of configuration space without sacrificing the Boltzmann sampling. The method targets specific degrees of freedom in the potentialenergy function. Without targeting, the scaled surface may be sufficiently enlarged relative to the original surface that the scheme would become computationally inefficient. An advantage of the reweighting scheme is its focus on the potential energy; thus, the nature and sizes of the barriers are explicit.

We have shown, by the use of the simple double-well system, that targeted reweighting is a computationally efficient method to carry out the desired Boltzmann sampling. A criterion for scaling was obtained that essentially says to scale barriers with a factor $g$ such that $g V_{b} / D \sim O(1)$, which will make sure that many transitions occur, yet the exploration of the configuration space is not greatly increased rela- 
tive to no scaling. This procedure could be refined in various ways. For example, when the potential is quite asymmetric, a modification of the potential surface that is concentrated around the barrier region of the potential will prevent the higher-energy stable state from being so reduced in energy that it becomes extensively sampled. The same procedure would be useful when the surface does not rise steeply at the extremes. That would prevent an increase in the configuration space that would reduce the computational efficiency when the surface is flattened too much.

A Langevin dynamics scheme was introduced that guarantees that if the sampling is sufficient then it will be from the correct equilibrium distribution. This could potentially be used to do atomistic molecular dynamics as long as all that is desired is Boltzmann sampling, versus dynamical information. The equations of motion are integrated with a stochastic Runge-Kutta integrator whose error can be estimated and is certainly of higher order than the conventional integrators used in molecular-dynamics simulations.

The utility of targeted reweighting, where only the terms of the potential that are anticipated to be important are scaled, was demonstrated in the double-well model with different barriers. A much more complex model of a chain molecule parametrized by dihedral potentials and bead-bead interactions provides a severe test of both the reweighting scheme and the integration method. If all the terms in the potential were uniformly scaled, the $\alpha$-helical parts of the chain would most likely undergo transitions. While the reweighting step should restore the proper sampling, the computational efficiency will rapidly degrade because the space sampled will grow rapidly. By focusing the scaling of the potential on the loop dihedrals, the sampling of the desired configuration space was greatly enhanced, and structures that resemble a helix-turn-helix generated from the all $\alpha$-helical initial state.

The ability to use a larger time step in the Runge-Kutta integrator when applied to the chain model is compromised to a certain extent by the time scale mismatch between the repulsive part of the van der Waals and dihedral barrier terms. Thus, it is possible, even with initial conditions that avoid steric clashes, to generate large forces for certain configurations. Adaptive step size Runge-Kutta methods are available that could mitigate the effect. ${ }^{46}$ However, these only apply to nonstochastic equations of motion. Developing high-order adaptive Runge-Kutta methods for the Langevin equation used in this work would be a useful improvement that we are currently investigating.

Finally, we note that the challenge for targeted reweighting is to decide which terms in the potential to scale. For example, in a protein, barriers to side-chain rotations must arise from van der Waals and electrostatic interactions with relatively close residues. It is possible to scale selectively some of these interactions but, clearly, the selection of what to scale is a nontrivial issue.

\section{ACKNOWLEDGMENTS}

One of the authors (R.I.C.) acknowledges financial support of the National Institutes of Health (GM47274). Another author (M.M.) acknowledges a travel grant awarded by the Secretaria de Estado de Educación y Universidades of Spain, and the Ministerio de Educación y Ciencia of Spain (project BFM2002-03822).

${ }^{1}$ A. McCammon and S. C. Harvey, Dynamics of Proteins and Nucleic Acids (Cambridge University Press, Cambridge, 1987).

${ }^{2}$ C. L. I. Brooks, M. Karplus, and B. M. Pettitt, Adv. Chem. Phys. 71, 259 (1988).

${ }^{3}$ J. D. Bryngelson, J. N. Onuchic, N. D. Socci, and P. G. Wolynes, Proteins: Struct., Funct., Genet. 21, 167 (1995).

${ }^{4}$ P. G. Wolynes, J. N. Onuchic, and D. Thirumalai, Science 267, 1619 (1995).

${ }^{5}$ C. D. Snow, E. J. Sorin, Y. M. Rhee, and V. S. Pande, Annu. Rev. Biophys. Biomol. Struct. 34, 43 (2005).

${ }^{6}$ Q. Cui, G. H. Li, J. P. Ma, and M. Karplus, J. Mol. Biol. 340, 345 (2004).

${ }^{7}$ M. K. Gilson, J. A. Given, B. L. Bush, and J. A. McCammon, Biophys. J. 72, 1047 (1997).

${ }^{8}$ T. Simonson, G. Archontis, and M. Karplus, Acc. Chem. Res. 35, 430 (2002).

${ }^{9}$ S. S. Plotkin and J. N. Onuchic, Q. Rev. Biophys. 35, 111 (2002).

${ }^{10}$ G. M. Torrie and J. P. Valleau, Chem. Phys. Lett. 28, 578 (1974).

${ }^{11}$ D. Frenkel and B. Smit, Understanding Molecular Simulation: From Algorithms to Applications (Academic, San Diego, CA, 1996).

${ }^{12}$ P. Kollman, Chem. Rev. (Washington, D.C.) 93, 2395 (1993).

${ }^{13}$ T. Rodinger and R. Pomes, Curr. Opin. Struct. Biol. 15, 164 (2005).

${ }^{14}$ P. E. Smith and W. F. van Gunsteren, J. Phys. Chem. 98, 13735 (1994).

${ }^{15}$ B. J. Berne and J. E. Straub, Curr. Opin. Struct. Biol. 7, 181 (1997).

${ }^{16}$ P. Hänggi, P. Talkner, and M. Borkovec, Rev. Mod. Phys. 62, 251 (1990).

${ }^{17}$ D. S. Chekmarev, T. Ishida, and R. M. Levy, J. Phys. Chem. B 108, 19487 (2004)

${ }^{18}$ C. Bartels, M. Schaefer, and M. Karplus, J. Chem. Phys. 111, 8048 (1999).

${ }^{19}$ U. H. E. Hansmann and Y. Okamoto, J. Comput. Chem. 18, 920 (1997).

${ }^{20}$ J. G. Kim, Y. Fukunishi, and H. Nakamura, Chem. Phys. Lett. 392, 34 (2004).

${ }^{21}$ A. Mitsutake, U. H. E. Hansmann, and Y. Okamoto, J. Mol. Graphics Modell. 16, 226 (1998).

${ }^{22}$ Y. Okamoto, J. Mol. Graphics Modell. 22, 425 (2004).

${ }^{23}$ Y. Sugita and Y. Okamoto, Chem. Phys. Lett. 329, 261 (2000).

${ }^{24}$ C. J. Woods, J. W. Essex, and M. A. King, J. Phys. Chem. B 107, 13703 (2003).

${ }^{25}$ U. H. E. Hansmann, Comput. Phys. Commun. 122, 129 (1999).

${ }^{26}$ U. H. E. Hansmann, Eur. Phys. J. B 12, 607 (1999).

${ }^{27}$ W. Wenzel and K. Hamacher, Phys. Rev. Lett. 82, 3003 (1999).

${ }^{28}$ H. Merlitz, B. Burghardt, and W. Wenzel, Chem. Phys. Lett. 370, 68 (2003).

${ }^{29}$ D. Hamelberg, J. Mongan, and J. A. McCammon, J. Chem. Phys. 120, 11919 (2004)

${ }^{30}$ Z. H. Liu and B. J. Berne, J. Chem. Phys. 99, 6071 (1993).

${ }^{31}$ M. P. Allen and D. J. Tildesley, Computer Simulation of Liquids (Oxford University Press, Oxford, 1987).

${ }^{32} \mathrm{H}$. Risken, The Fokker-Planck Equation: Methods of Solution and Applications (Springer-Verlag, Berlin, 1984)

${ }^{33}$ R. Zwanzig, Nonequilibrium Statistical Mechanics (Oxford University Press, Oxford, 2001).

${ }^{34}$ E. Helfand, Bell Syst. Tech. J. 58, 2289 (1979).

${ }^{35}$ H. S. Greenside and E. Helfand, Bell Syst. Tech. J. 60, 1927 (1981).

${ }^{36}$ R. Zwanzig, A. Szabo, and B. Bagchi, Proc. Natl. Acad. Sci. U.S.A. 89, 20 (1992)

${ }^{37}$ K. A. Dill and H. S. Chan, Nat. Struct. Biol. 4, 10 (1997).

${ }^{38}$ J. Durup, J. Mol. Struct.: THEOCHEM 424, 157 (1998).

${ }^{39}$ S. Q. He and H. A. Scheraga, J. Chem. Phys. 108, 271 (1998).

${ }^{40}$ S. Q. He and H. A. Scheraga, J. Chem. Phys. 108, 287 (1998).

${ }^{41}$ J. Casado-Pascual, C. Denk, J. Gómez-Ordóñez, M. Morillo, and P. Hänggi, Phys. Rev. E 67, 036109 (2003). 
${ }^{42}$ C. Branden and J. Tooze, Introduction to Protein Structure (Garland, New York, 1999).

${ }^{43}$ C. R. Cantor and P. R. Schimmel, Biophysical Chemistry (Freeman, San Francisco, 1980).

${ }^{44}$ W. A. McLaughlin and H. M. Berman, J. Mol. Biol. 330, 43 (2003).
${ }^{45}$ S. C. Harrison and A. K. Aggarwal, Annu. Rev. Biochem. 59, 933 (1990).

${ }^{46}$ W. H. Press, S. A. Teukolsky, W. T. Vetterling, and B. P. Flannery, Numerical Recipes in FORTRAN (Cambridge University Press, Cambridge, England, 1992). 